\title{
Reliability testing of a modified MISTELS score using a low-cost trainer box
}

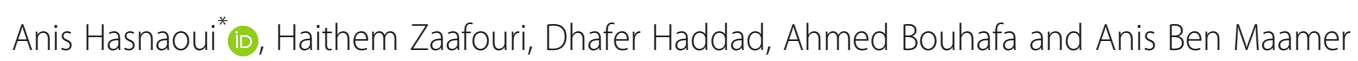

\begin{abstract}
Background: Training programs such as the fundamentals of laparoscopic surgery (FLS) that are based on simulation are being currently used in several western countries. FLS allows skill acquisition and evaluation of competency in laparoscopic surgery. On the practical side, evaluation is determined by the MISTELS metrics (MISTELS is the acronym for the McGill inanimate system for training and evaluation of laparoscopic skills). This training program may be modified so that it can be implemented in countries with limited resources using a low-cost trainer box. Would the use of a low-cost trainer box alter the reliability of the MISTELS score?

Objective of study: The aim of the study was to evaluate the reliability of a modified MISTELS using a low-cost trainer box.

Methods: It was a prospective study carried out at Habib Thameur hospital in Tunis (Tunisia), between April 2016 and August 2016. The study involved residents from different surgical specialties in the departments of general surgery and paediatric surgery of the hospital during 2015 and 2016.

This study assessed the reliability of a modified MISTELS system (Only three tasks were performed out of the five tasks used in the original MISTELS system). Evaluation was based on Cronbach's alpha and intraclass correlation coefficients (ICC).

A low-cost trainer box was designed and constructed. The residents included in the study performed three series of three tasks using this trainer box. The first series was scored by two trained raters to evaluate inter-rater reliability. The two-other series were successively performed to evaluate test-retest reliability.

Results: The internal consistency, assessed by Cronbach's alpha, was at 0.929 which is an acceptable score. As for inter-rater and test-retest reliabilities that were assessed by ICCs, they yielded excellent scores that were at 1 and 0. 95 (95\% Cl, 0.891-0.978) respectively.
\end{abstract}

Conclusions: The reliability of a modified MISTELS is not altered by the use of a low-cost trainer box. The score of the modified MISTELS is a reliable score for evaluating technical skills of surgical residents using a low-cost trainer box.

Keywords: Training, Evaluation, Simulation, FLS, MISTELS, Reliability, Low-cost, Trainer box

\section{Background}

In Tunisia, acquisition of technical skills in surgery is based on observation of senior surgeons in the operating room. As for assessment, it is based on evaluation of theoretical knowledge, leaving out the practical side.

In some western countries, simulation has become an essential part of the training program for young surgical residents in laparoscopic surgery. The fundamentals of

\footnotetext{
* Correspondence: hasnaouianis001@gmail.com

Department of General Surgery, Habib Thameur Hospital, Ali Ben Ayed,

Street 2037 Montfleury, Tunis, Tunisia
}

laparoscopic surgery (FLS) is a program based on the McGill inanimate system for training and evaluation of laparoscopic skills (MISTELS) [1] which allows better acquisition of theoretical and practical skills in laparoscopic surgery as well as evaluation of these skills [2-4]. On the practical side, participants perform five tasks using a trainer box, to improve the basic skills of laparoscopic surgery. Once the tasks performed, a score based on evaluation of the time of execution and precision is given: the score of MISTELS.

(c) The Author(s). 2019 Open Access This article is distributed under the terms of the Creative Commons Attribution 4.0 International License (http://creativecommons.org/licenses/by/4.0/), which permits unrestricted use, distribution, and reproduction in any medium, provided you give appropriate credit to the original author(s) and the source, provide a link to the Creative Commons license, and indicate if changes were made. The Creative Commons Public Domain Dedication waiver (http://creativecommons.org/publicdomain/zero/1.0/) applies to the data made available in this article, unless otherwise stated. 
This program may be modified using a low-cost trainer box and consumables, so that it can be used in countries with limited resources like ours. But would these modifications alter the reliability of the score of MISTELS.

The aim of this study was to assess the reliability of a modified MISTELS score, based on the performance of three tasks instead of five, using a low-cost trainer box, and to discuss the importance of low-cost simulators in surgical training.

\section{Methods}

It was a prospective study carried out at Habib Thameur hospital in Tunis (Tunisia) between April 2016 and August 2016. The study included surgical residents of different surgical specialties in the departments of general surgery and paediatric surgery during the period 20152016. We excluded from the study the trainees who couldn't participate or complete all the tasks.

Evaluation of the reliability was based on Cronbach's alpha [5] which assessed the internal consistency of the scores assigned to different tasks in a single series, and the intraclass correlation coefficient (ICC) [6] which assessed the correlation between the score of different series.

Using a linear folder, we shaped rectangular plates of acrylic glass (Fig. 1) to obtain a trainer box measuring $30 * 37 * 23.5 \mathrm{~cm}$ (width*length*height). A high definition webcam (Logitech C920 HD Pro) was used in the study. To fix it on the trainer box, we used a holder of a desk lamp. Two LED lamps provided lighting. The cost of the box was estimated at 500 Tunisian Dinars (Which is the equivalent of $\$ 165$ or $£ 132.33$ using the exchange rate on 06th January 2019), not counting the cost of the laparoscopic forceps. A portable computer was used to obtain the images from the webcam. The streaming of the images was obtained by the multimedia reader VLC 2.2.4 which is a freeware. Laparoscopic surgical instruments (Maryland forceps, a grasper, scissors and a needle holder) were used (Fig. 2). To videotape the performed tasks, we used the camera of a smartphone facing the central opening of the trainer box.

The residents were scored on their performance of three tasks from the MISTELS program: peg transfer, precision cutting and suture with intra-corporeal knot (Fig. 3).

Each resident had a simulation session comprising 3 phases: briefing, simulation and debriefing.

During briefing, we explained to the residents the different aspects of the session: progress, technique and pointers. After the briefing, each resident performed three series of three tasks (peg transfer, precision cutting and suture with intra-corporeal knot) as stated in the literature [1]. The two other tasks of the MISTELS program (Ligating loop and suture with extra-corporeal knot) were not performed due to financial constraints.
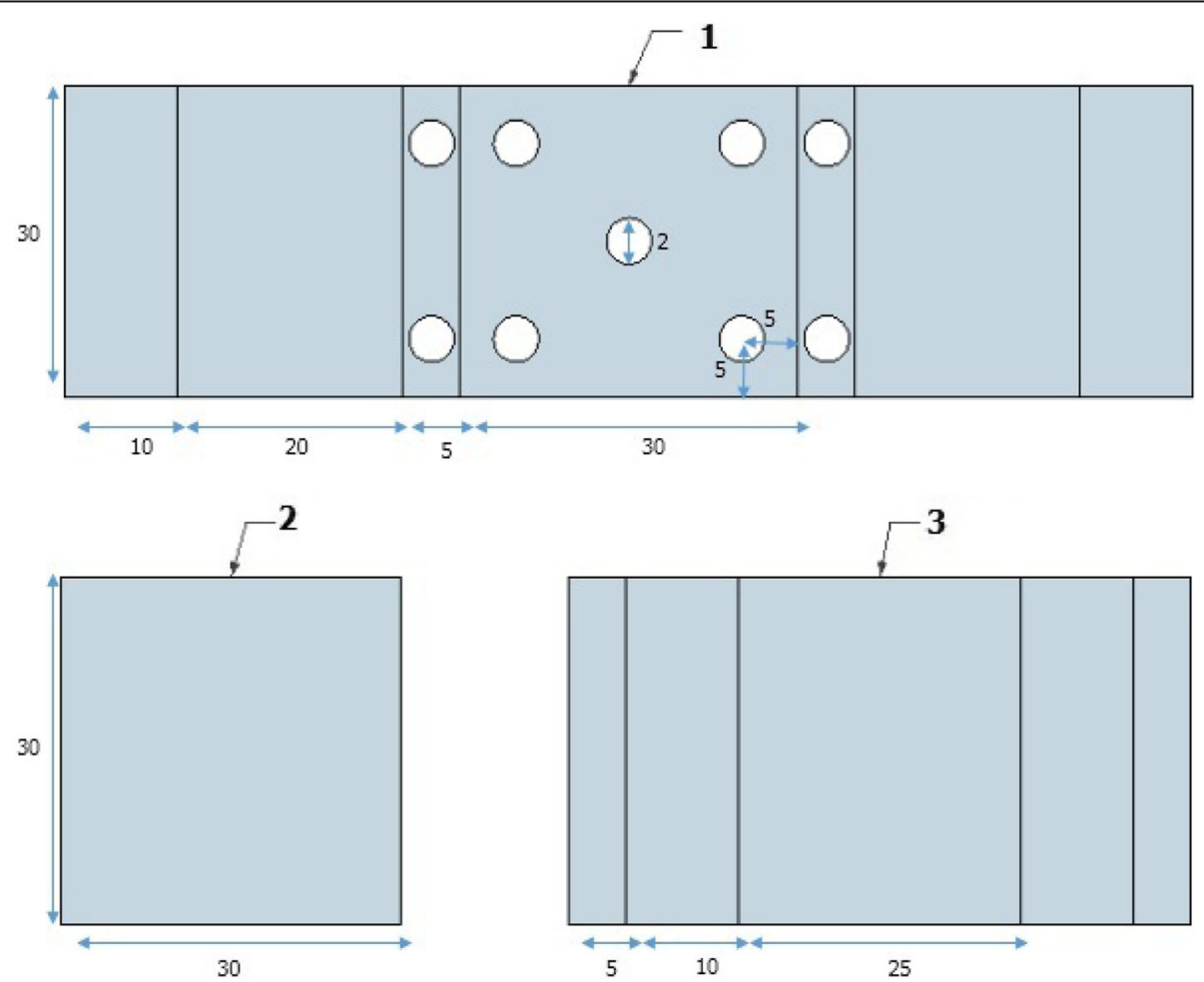

Fig. 1 Measurements in $\mathrm{cm}$ of the plates and folding lines 


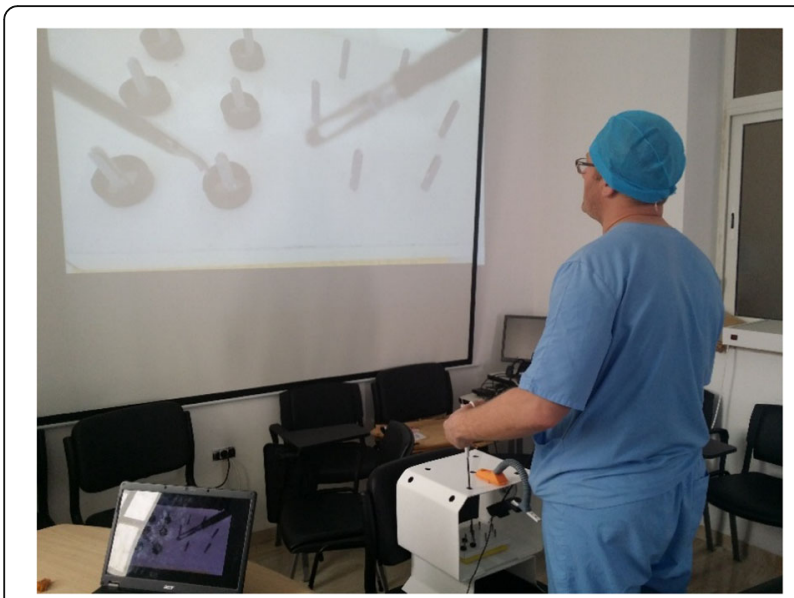

Fig. 2 Images shown in real time onto the screen of the computer and the wall screen

Debriefing aimed at underlining the positive and negative aspects of the session, laying down the acquired knowledge and bringing out the possible improvements that should be made.

Assessment of the performance of the residents was done in conformity with the data described in the literature [1]. The score for each task was normalized by dividing the score obtained by a predetermined standard value that was derived from the maximum score achieved by a chief resident for that task and then multiplying by 100 .

For the first series of task, calculation of the scores was done by two raters to study the reliability between two raters. The first rater scored the performance on the spot at the end of each task. The second rater, who was blinded, scored the performances based on videotapes and tools kept intact after the tasks. The two-other series were successively performed and were scored by a single rater to study test-retest reliability. Thus, we obtained four scores for each resident (rater 1, rater 2, test and retest scores).
For statistical analysis, we used the SPSS version 21.0.0.0 software. Quantitative variables were expressed in averages. Internal consistency was determined using Cronbach's alpha. Internal consistency was determined for the 4 series (rater 1, rater 2, test, retest) using the basic scores obtained for the three tasks (peg transfer, precision cutting and suture with intra-corporeal knot) in each series. The deletion impact of each of the three tasks on the internal consistency was evaluated in the last series (retest). Test-retest and inter-rater reliabilities were determined by the ICC. Each ICC was calculated using a two-way mixed model with absolute agreement and a $95 \%$ confidence interval.

\section{Results}

All the results of the study are presented in Additional file 1 . The study involved 26 residents having different surgical specialties. One of the residents was excluded from the study because he didn't perform all the tasks. Distribution of residents by specialty was as follows: fourteen residents of general surgery, five of paediatric surgery, two of urology, two surgical residents requesting for an equivalent rating of their diploma, a resident of surgical oncology and a resident of gynaecology. The residents were aged 30.08 years on average (range 2738 ). Nineteen of the residents had already had some experience in laparoscopic surgery.

To assess the internal consistency of the MISTELS score, we calculated Cronbach's alpha coefficient for each of the series (rater 1 , rater 2, test, retest). The obtained Cronbach's alpha coefficients were all acceptable. Table 1 shows the correlation of each task with the total score achieved in the last series (retest), as well as the deletion impact of each task on Cronbach's alpha coefficient.

Scores of each task had a high correlation with the total score. Deletion of the second exercise (precision cutting)

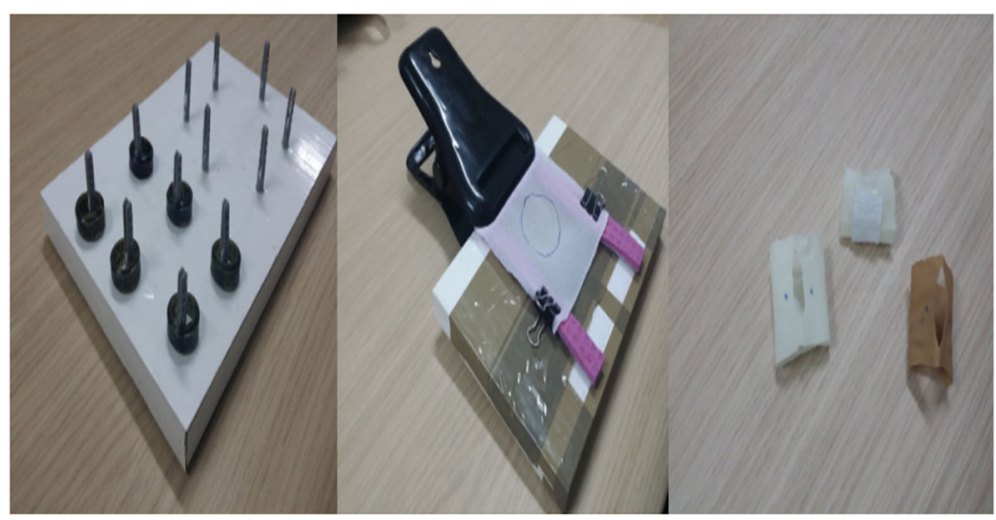

Fig. 3 Materials used during the performance of the MISTELS tasks 
Table 1 Correlation between the score of each task and the total score and impact of deletion of each task on Cronbach's alpha and thus on internal consistency (Scores obtained in the series of retest)

\begin{tabular}{lll}
\hline Tasks & $\begin{array}{l}\text { Correlation with } \\
\text { the total score }\end{array}$ & $\begin{array}{l}\text { Cronbach's alpha in case } \\
\text { of deletion of the exercise }\end{array}$ \\
\hline Peg transfer & 0.972 & 0.832 \\
Precision cutting & 0.894 & 0.951 \\
intra-corporeal knot & 0.952 & 0.889 \\
\hline
\end{tabular}

would slightly improve Cronbach's alpha (0.951). Deletion of the two other tasks would, on the other hand, lower it.

Inter-rater reliability was evaluated using the ICC. This coefficient was equal to one which confirmed the low variability between the scores of two raters (Table 2).

The total scores granted by each rater are represented by curves in Fig. 4 . Both curves are matching which confirms the low variability between raters.

The test-retest reliability was excellent with an ICC of 0.95 (95\% CI, 0.891-0.978) (Table 3).

The scores granted in the test and retest are shown in Fig. 5. The curves are not totally matching which indicates a difference, minimal as it may seem, in the scores obtained in the test and retest series.

\section{Discussion}

\section{Reliability testing using a low-cost trainer box}

Using a low-cost trainer box, we found that the modified MISTELS score is a reliable score for assessing competency in laparoscopic surgery.

Reliability refers to the consistency, stability and precision of a test results [7]. It is important to evaluate the reliability for two main reasons. Firstly, the necessity to establish evidence for reliability is driven by errors that characterize all instruments of measurement. The impact of these errors on the scores of a test is unpredictable for these errors occur at random. Using an unreliable test is like giving useless random scores to the participants in this test. Secondly, assessment of reliability always precedes the study of validity. In fact, an effective test should be reliable and valid [8]. Proving the

Table 2 Internal consistency assessed by Cronbach's alpha and inter-rater reliability assessed by ICC of the modified MISTELS score

\begin{tabular}{|c|c|c|c|c|c|}
\hline \multirow[t]{2}{*}{ Tasks } & \multicolumn{2}{|c|}{ Rater 1} & \multicolumn{2}{|l|}{ Rater 2} & \multirow[t]{2}{*}{$I C C^{b}$} \\
\hline & Mean & $S D^{a}$ & Mean & SD & \\
\hline Peg transfer & 32.49 & 30.22 & 32.38 & 30.12 & 1 \\
\hline Precision cutting & 20.80 & 24.61 & 20.63 & 24.62 & 1 \\
\hline intra-corporeal knot & 27.42 & 29.58 & 27.5 & 29.70 & 1 \\
\hline Total score & 80.72 & 81.14 & 80.52 & 81.02 & 1 \\
\hline Cronbach's alpha & 0.955 & 0.953 & & & \\
\hline
\end{tabular}

${ }^{a} S D$ Standard deviation, ${ }^{b}$ ICC Intraclass correlation coefficient validity of a test is a long process which requires several steps and experiments. Before embarking on such a procedure, we should first make sure that the measures are reliable. Otherwise, the results will be false.

The study of reliability comprises an assessment of the internal consistency based on the calculation of Cronbach's alpha and assessment of the inter-rater and test-retest reliabilities by calculating the ICC.

\section{Internal consistency}

The internal consistency of a test ensures that all the components of a score are measuring the same thing [9]. It is considered satisfactory if alpha varies between 0.7 and 1 [9]. In this study, Cronbach's alphas in the different series were superior to 0.9 which means that they were acceptable [9]. This suggests that the three performed tasks in our study measured repeatedly and constantly the resident's competency in laparoscopic surgery and supports the use of the total score as a one-dimensional scale. By reconsidering the formula of this coefficient, we remark that the internal consistency is proportional to the length of the test [10]. However, in our study, we used three tasks out of the five tasks in the original MISTELS score. Spearman-Brown prophecy formula $[11,12]$ statistically predicts reliability of a modified test insofar as the added or subtracted items are qualitatively equivalent to those comprised in the initial test [13]. Based on this formula, Cronbach's alpha coefficients would have better values than those found in our study.

In the literature, we found only one study that assessed the reliability of the MISTELS score [14]. It was carried out by Vassiliou et al. In their study involving 12 subjects, the best value of Cronbach's alpha was achieved during the test. It was equal to 0.86 , which is an acceptable value [9]. Cronbach's alpha coefficients in our study were slightly superior to those reported in the literature. This may be explained by the scores obtained by the inexperienced residents in our study, those who obtained a score of zero in all the exercises of a given series. The number of these residents varied between 2 (In the series of retest) and 10 (In the series of scores given by both raters). If we delete the results of the two residents who obtained a score of zero in all the retest tasks, the Cronbach's alpha coefficient in the series of retest would be 0.899 . This is an acceptable value which is in accordance with the value reported in the literature.

\section{Inter-rater and test-retest reliabilities}

In the evaluation of the inter-rater reliability, the errors of measurement may be due to the different raters using different methods. In our study, we didn't expect a significant difference between the scores given by the two raters for a same test, for measurements of time and 


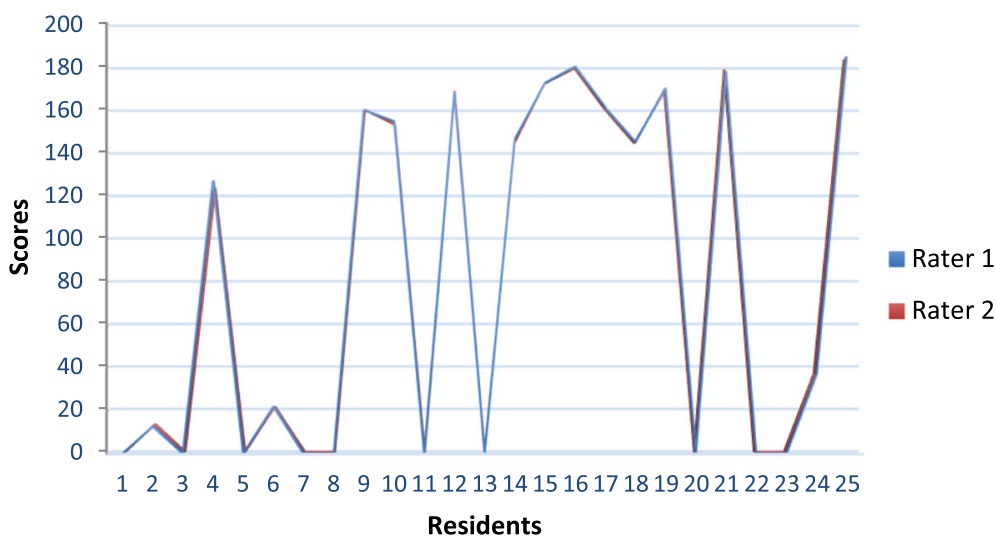

Fig. 4 Modified MISTELS scores given to residents by two raters

precision were standardized. ICC used to assess inter-rater reliability was equal to one, confirming the low variability between raters. In the study of Vassiliou et al., the ICC for inter-rater reliability was at 0.998 (95\% CI, 0.985-1). This result was in accordance with our result.

As for the assessment of test-retest reliability, errors of measurement can be grouped into three categories: Errors associated with the raters, errors associated with the equipment used for the test and errors associated with the residents themselves (Different performances due to the effect of practice or to fatigue, distraction and frustration). Errors associated with the raters and with the used equipment were limited by standardizing methods of measurement by the raters and using predefined measurements for the equipment used in the tasks. As for the performance of each resident, it may improve especially for beginners (MISTELS is a training program that is supposed to improve competency in laparoscopic surgery $[1,15,16])$. Or it may deteriorate due to fatigue or frustration (Hence the importance of motivating the residents). To minimize these errors, the test and retest were performed successively without free interval. Nevertheless, two novice residents had a score of zero in the test but improved their score in the retest. ICC of the test-retest was excellent with a value of 0.95 (95\%

Table 3 Internal consistency assessed by Cronbach's alpha and test-retest reliability assessed by ICC of the modified MISTELS score

\begin{tabular}{lllllll}
\hline Tasks & \multicolumn{1}{l}{ Test } & & & Retest & & ICC $^{\mathrm{b}}$ \\
\cline { 2 - 3 } & Mean & $\mathrm{SD}^{\mathrm{a}}$ & & Mean & $\mathrm{SD}$ & \\
\hline Peg transfer & 33.55 & 28.72 & & 36.26 & 29.23 & 0.952 \\
Precision cutting & 26.69 & 24.09 & 32.96 & 23.07 & 0.769 \\
intra-corporeal knot & 31.45 & 32.47 & 28.96 & 31.17 & 0.902 \\
Total score & 91.69 & 80.65 & & 98.18 & 78.70 & 0.950 \\
Cronbach's alpha & 0.933 & & & 0.929 & & \\
\hline
\end{tabular}

${ }^{\mathrm{a} S D}$ Standard deviation, ${ }^{\mathrm{b}} \mathrm{ICC}$ Intraclass correlation coefficient
CI, 0.891-0.978). In the study carried out by Vassiliou et al., the ICC of the test-retest was slightly inferior to the value reported in our study. It was equal to 0.892 (95\% CI, 0.665-0.968) [14]. According to Vassiliou et al., ICC of the test-retest was inferior to the ICC of the inter-rater reliability due to the effect of practice on the performance of the trainees. In fact, it was found that practice using MISTELS trainer improved the score of trainees even after a single training session $[1,15,16]$. This improvement was more marked in the less experienced residents. To decrease the effect of practice on performance, Vassiliou included the residents who had achieved a total score (including the five tasks of MISTELS) of 230. In addition, the test and retest series were performed successively. In our study, the effect of practice on performance was not so well marked. The ICC of the test-retest in our study was higher than the ICC reported in Vassiliou's study; which raises the question of the effectiveness of our model in comparison to the original model of MISTELS. The answer to this question would probably require further research study to test the validity of our model.

The importance of low-cost simulators in surgical training Simulation has become the corner stone of surgical training in many developed countries. Many scores have been introduced using different models ranging from low fidelity and low-cost trainers to high fidelity simulation trainers and animal models. This wide range raised the debate of the most effective and cost/effective model.

Many studies proved the effectiveness of low-cost trainers in surgical training [17] and their reliability in the evaluation of training [18]. Chandrasekera et al. used a randomised blinded study to compare the effectiveness of a cheap training model using a cardboard box and a conventional video-laparoscopy trainer [19]. The authors found no significant difference between the two models. In their study, the cost of the conventional trainer was 


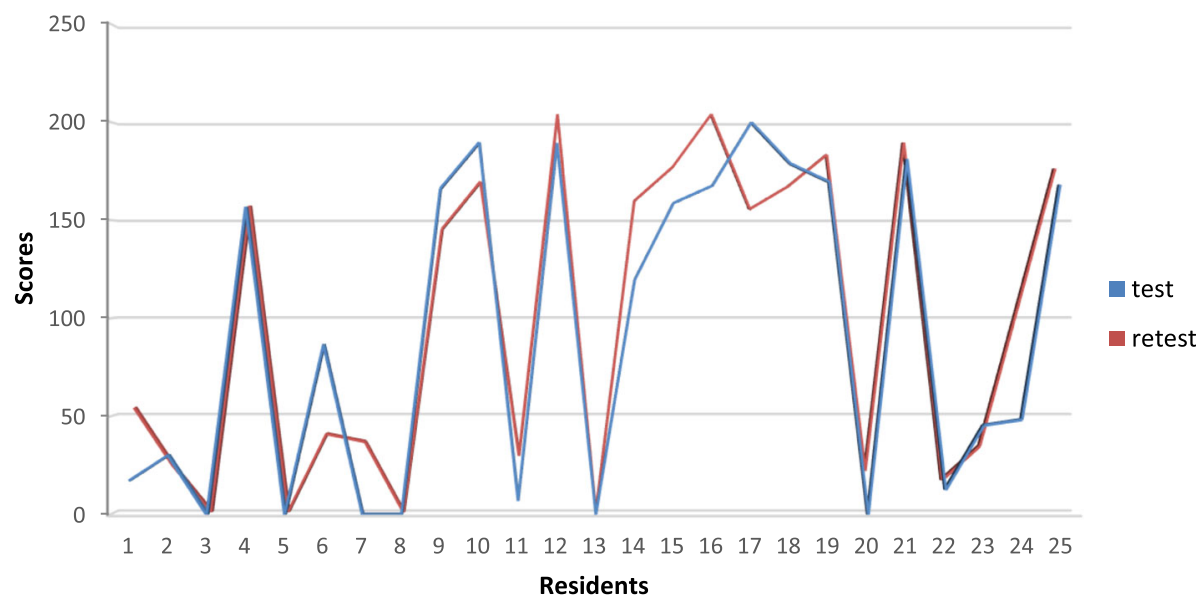

Fig. 5 Modified MISTELS scores given to residents in the test and the retest series by a single rater

30.000 euros. Thus, they concluded that the cardboard box is more efficient (Efficiency as the relationship between cost and effectiveness) in the training. Sandberg et al. used a randomised controlled trial to validate a non-anatomic and low-cost model for arthroscopy training [20]. This study concluded that the model is an effective knee arthroscopy trainer that may decrease the learning curve without significant cost.

It is obvious that the cost is the main advantage in using low-cost trainers. It offers novice surgeons the possibility of more regular training, even at home. In a systematic review of low-cost laparoscopic simulators, Mimi M. Li et al. identified 73 unique simulators $(60$ non-commercial, 13 commercial) [21]. The cost ranged from $£ 3$ to $£ 216$ for non-commercial and $£ 60$ to $£ 1007$ for commercial simulators. Forty-five per cent (27) of non-commercial simulators in this review were not subject to any validation test [21]. The cost of our model (Hardware and labor) was $£ 132.33$ using the exchange rate on 06th January 2019. We should mention that the cost of the webcam was around $£ 85$. Furthermore, the use of low-cost consumables, like in our study, could drastically reduce the cost of training. In a cost comparison study between standard FLS equipment and low-cost equipment, Franklin BR et al. found that the use of low-cost equipment results in significant financial savings and that a five-resident program will save approximately $\$ 8500$ annually [22].

Thus, low-cost simulators are more efficient than high fidelity simulators, offering equivalent training results with lower cost. On the other hand, high fidelity simulators and animal models offer more realistic situations and variability for the trainees to deal with. Therefore, we encourage the conjunction of the different simulators for a complete training when it is possible.

\section{Conclusions}

Even though additional evidence is still required, the value of simulation in laparoscopic surgery can by no means be ignored or underestimated [23]. Simulation should be offered to every resident as part of their training program. The score of the modified MISTELS is a reliable score for evaluating the technical skills of surgical residents using the trainer box that we have constructed. Keeping in mind the financial constraints in countries with limited resources, we consider that implementing FLS using low-cost materials can provide a solution to this problem that hinders the training of young residents.

\section{Additional file}

Additional file 1: Results of the study containing the raw data used in the study. (XLSX $11 \mathrm{~kb})$

Abbreviations

FLS: Fundamentals of laparoscopic surgery; ICC: Intraclass correlation coefficient; MISTELS: McGill inanimate system for training and evaluation of laparoscopic skills

\section{Acknowledgements}

Not applicable.

Funding

No funding was received.

Availability of data and materials

Data and materials are available at the request of the readers. Requests for data should be addressed to Hasnaoui Anis.

\section{Authors' contributions}

$\mathrm{HA}$ and $\mathrm{ZH}$ designed the study. $\mathrm{HA}, \mathrm{ZH}$ and $\mathrm{HD}$ acquired the data. $\mathrm{HD}$ and $\mathrm{HA}$ analysed and interpreted the data. $\mathrm{HA}$ and $\mathrm{ZH}$ drafted the manuscript. $B A$ and $B M A$ revised the final version of the manuscript. All authors read and approved the final manuscript. 


\section{Ethics approval and consent to participate}

The study was approved by the Ethical Review Board of Habib Thameur Hospital. Written consent was obtained from all participants.

\section{Consent for publication}

Not applicable.

\section{Competing interests}

The authors declare that they have no competing interests.

\section{Publisher's Note}

Springer Nature remains neutral with regard to jurisdictional claims in published maps and institutional affiliations.

Received: 6 March 2018 Accepted: 24 April 2019

Published online: 06 May 2019

\section{References}

1. Derossis AM, Fried GM, Abrahamowicz M, et al. Development of a model for training and evaluation of laparoscopic skills. Am J Surg. 1998;175:482-7.

2. Fried GM, Feldman LS, Vassiliou MC, et al. Proving the value of simulation in laparoscopic surgery. Ann Surg. 2004;240(3):518-25

3. Ritter EM, Scott DJ. Design of a proficiency-based skills training curriculum for the fundamentals of laparoscopic surgery. Surg Innov. 2007;14(2):107-12.

4. Scott DJ, Ritter EM, Tesfay ST, et al. Certification pass rate of $100 \%$ for fundamentals of laparoscopic surgery skills after proficiency-based training. Surg Endosc. 2008;22(8):1887-93.

5. Cronbach L. Coefficient alpha and the internal structure of tests, Psychometrika. 1951;16:297-302.

6. Shrout PE, Fleiss JL. Intraclass correlations: uses in assessing reliability. Psychol Bull. 1979;86:420-8.

7. Streiner D, Norman G. Health measurement scales: a practical guide to their development and use. New York: Oxford University Press; 1996.

8. Gall M, Borg W, Gall J. Educational research: an introduction. 8th ed. New York: Pearson; 2006.

9. Bland JM Altman DG. Cronbach's alpha. Br Med J. 1997:314:572.

10. Gary GM. Handbook of psychological assessment. 4th ed. New Jersey: Wiley; 2003. p. 16

11. Spearman C. Correlation calculated from faulty data. Br J Psychol. 1910;3(3): 271-95.

12. Brown W. Some experimental results in the correlation of mental abilities. $\mathrm{Br}$ J Psychol. 1910;3(3):296-322.

13. Allison PD. A simple proof of the spearman-brown formula for continuous test lengths. Psychometrika. 1976;41(1):135-6.

14. Vassiliou M, Ghitulescu G, Feldman L, et al. The MISTELS program to measure technical skill in laparoscopic surgery. Surg Endosc. 2006;20(5):744-7.

15. Derossis AM, Bothwell J, Sigman HH, et al. The effect of practice on performance in a laparoscopic simulator. Surg Endosc. 1998;12:1117-20.

16. Fried GM, Derossis AM, Bothwell J, et al. Comparison of laparoscopic performance in vivo with performance measured in a laparoscopic simulator. Surg Endosc. 1999;13:1077-82.

17. Brusalis CM, Lawrence JTR, Ranade SC, et al. Can a novel, low-cost simulation model be used to teach anterior cruciate ligament graft preparation? Pediatr Orthop. 2017:37(4):277-81.

18. Adrales $G L$, Chu UB, Witzke DB, et al. Evaluating minimally invasive surgery training using low-cost mechanical simulations. Surg Endosc. 2003;17(4): 580-5.

19. Chandrasekera SK, Donohue JF, Orley D, et al. Basic laparoscopic surgical training: examination of a low-cost alternative. Eur Urol. 2006;50:1285-91.

20. Sandberg RP, Sherman NC, Latt LD, et al. Cigar box arthroscopy: a randomized controlled trial validates nonanatomic simulation training of novice arthroscopy skills. Arthroscopy. 2017;33(11):2015-23.

21. Li MM, George J. A systematic review of low-cost laparoscopic simulators. Surg Endosc. 2017;31:38-48.

22. Franklin BR, Placek SB, Wagner MD, et al. Cost comparison of fundamentals of laparoscopic surgery training completed with standard fundamentals of laparoscopic surgery equipment versus low-cost equipment. J Surg Educ. 2017;74(3):459-65

23. Gaba DM The future vision of simulation in health care Qual Saf Health Care. 2004;13(1):2-10.

Ready to submit your research? Choose BMC and benefit from:

- fast, convenient online submission

- thorough peer review by experienced researchers in your field

- rapid publication on acceptance

- support for research data, including large and complex data types

- gold Open Access which fosters wider collaboration and increased citations

- maximum visibility for your research: over $100 \mathrm{M}$ website views per year

At $\mathrm{BMC}$, research is always in progress.

Learn more biomedcentral.com/submissions 